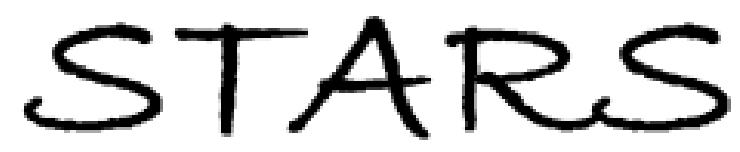

University of Central Florida

STARS

$1-1-2010$

\title{
Thermally controlled preferential molecular aggregation state in a thiacarbocyanine dye
}

\author{
Rémy Passier \\ University of Central Florida \\ James P. Ritchie \\ University of Central Florida \\ Carlos Toro \\ University of Central Florida \\ Carlos Diaz \\ University of Central Florida \\ Artëm E. Masunov \\ University of Central Florida \\ Find similar works at: https://stars.library.ucf.edu/facultybib2010 \\ University of Central Florida Libraries http://library.ucf.edu \\ See next page for additional authors \\ This Article is brought to you for free and open access by the Faculty Bibliography at STARS. It has been accepted for \\ inclusion in Faculty Bibliography 2010 s by an authorized administrator of STARS. For more information, please \\ contactSTARS@ucf.edu.
}

\section{Recommended Citation}

Passier, Rémy; Ritchie, James P.; Toro, Carlos; Diaz, Carlos; Masunov, Artëm E.; Belfield, Kevin D.; and Hernandez, Florencio E., "Thermally controlled preferential molecular aggregation state in a thiacarbocyanine dye" (2010). Faculty Bibliography 2010s. 630.

https://stars.library.ucf.edu/facultybib2010/630

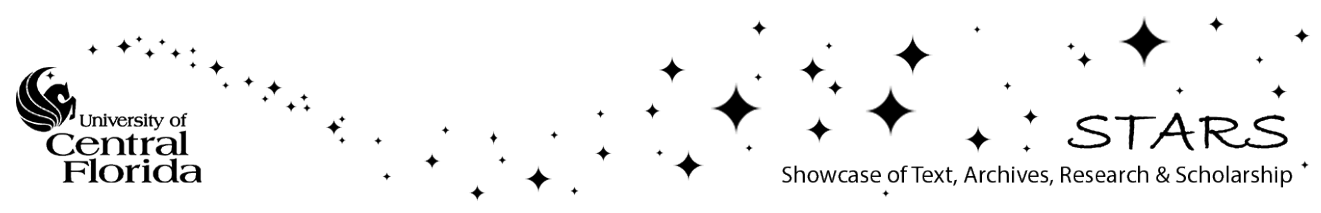




\section{Authors}

Rémy Passier, James P. Ritchie, Carlos Toro, Carlos Diaz, Artëm E. Masunov, Kevin D. Belfield, and Florencio E. Hernandez 


\section{Thermally controlled preferential molecular aggregation state in a thiacarbocyanine dye}

Cite as: J. Chem. Phys. 133, 134508 (2010); https://doi.org/10.1063/1.3483896

Submitted: 28 May 2010 . Accepted: 06 August 2010 . Published Online: 07 October 2010

Rémy Passier, James P. Ritchie, Carlos Toro, Carlos Diaz, Artëm E. Masunov, Kevin D. Belfield, and Florencio E. Hernandez

ARTICLES YOU MAY BE INTERESTED IN

Exciton transport in thin-film cyanine dye J-aggregates

The Journal of Chemical Physics 137, 034109 (2012); https://doi.org/10.1063/1.4732122

Annihilation of singlet excitons in J aggregates of pseudoisocyanine (PIC) studied by pico- and subpicosecond spectroscopy

The Journal of Chemical Physics 89, 2754 (1988); https://doi.org/10.1063/1.455027

Cyanine dye acting both as donor and acceptor in heterojunction photovoltaic devices

Applied Physics Letters 82, 3788 (2003); https://doi.org/10.1063/1.1579133

Where in the world is AIP Publishing? Find out where we are exhibiting next 


\title{
Thermally controlled preferential molecular aggregation state in a thiacarbocyanine dye
}

\author{
Rémy Passier, ${ }^{1}$ James P. Ritchie, ${ }^{2}$ Carlos Toro, ${ }^{1}$ Carlos Diaz, ${ }^{1}$ Artëm E. Masunov, ${ }^{1,2,3}$ \\ Kevin D. Belfield, ${ }^{1, a)}$ and Florencio E. Hernandez $z^{1, a), b)}$ \\ ${ }_{1}^{1}$ Department of Chemistry, University of Central Florida, P.O. Box 162366, Orlando, Florida 32816-2366, \\ USA \\ ${ }^{2}$ Nanoscience Technology Center, University of Central Florida, P.O. Box 162366, Orlando, Florida 32826, \\ USA \\ ${ }^{3}$ Department of Physics, University of Central Florida, P.O. Box 162366, Orlando, Florida 32816-2366, \\ USA
}

(Received 28 May 2010; accepted 6 August 2010; published online 7 October 2010)

\begin{abstract}
Herein we report the experimental and theoretical study of the temperature dependence of a thiacarbocyanine dye in its monomer, H- and J-aggregates states. We demonstrate the ability to control the ratio of monomer, $\mathrm{H}$ - and/or J-aggregates with heat. We link such a control to the conformation dependence of the molecule. An alternative way to gain access to the dominating species without changing the concentration as a complete switching mechanism between all the present species is proposed. The results presented in this work lead to a better understanding of thiacarbocyanine dye's behavior. () 2010 American Institute of Physics. [doi:10.1063/1.3483896]
\end{abstract}

\section{INTRODUCTION}

Molecular aggregation such as the one observed in cyanine dyes has been known for more than 50 years. ${ }^{1}$ Aggregates are defined in function of their stacking geometries that typically result in a blue (H-aggregates) or red (J-aggregates) spectral shift with respect to the monomer absorption band. ${ }^{2}$ The observed spectral shift depends on the aggregate preferential arrangement and is typically used to identify the type of aggregate present in the solution under certain conditions. During the past decades, an abundant number of fundamental and applied studies have demonstrated the potential applications of these entities to the field of photography, ${ }^{3}$ sensors, ${ }^{4}$ photoconductors, ${ }^{5,6}$ biology, ${ }^{7}$ medicine, ${ }^{8}$ and nanotechnology through the formation of self-assembled materials. ${ }^{9}$ The versatility of cyanine dyes relates to their ability to interact as liquid or solid state devices. ${ }^{10-12}$

After Franck and Teller ${ }^{13}$ demonstrated the exciton theory on the basis of Frenkel's work, ${ }^{14}$ experiments were decisive in understanding the effect that pressure, ${ }^{15}$ substituents, ${ }^{16}$ and concentration ${ }^{17}$ have on the aggregation mechanism of this family of molecules. Other studies, which performed to elucidate the channels of formation and organization of molecular aggregates in the solution, have concentrated on the impact of adding proteins ${ }^{18}$ and surfactants ${ }^{19,20}$ to the mixtures. Additionally, Van der Auweraer and co-workers ${ }^{21}$ have reported the relevance of considering the influence of molecular structure in the aggregation properties of cyanine dyes in Langmuir films. Interesting to highlight is the fact that most of the studies reported so far focus on the typical narrow band located at approximately $600 \mathrm{~nm}$, corresponding to the well known J-aggregate (end-

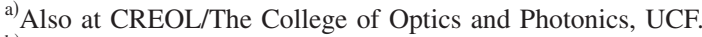

b) Author to whom correspondence should be addressed. Electronic mail: florenzi@mail.ucf.edu.
}

to-end aggregation of monomer units), for which a mechanism of growth from monomers and dimers has been proposed. $^{22}$

During the past 2 decades, the J-aggregates have found very interesting applications in nonlinear optics, ${ }^{23-26}$ mainly because of the substantial enhancement effect of these properties due to the excitonic coupling present in these structures. ${ }^{27}$ Although much is already known about the aggregates, there is still a great need for understanding more in depth the reduction of photobleaching ${ }^{28}$ and the mechanisms of stabilization of big aggregates in the solutions along with the minimization of scattering due to inhomogeneities in the medium at high concentration. ${ }^{29,30}$ In order to answer these questions, the scientific community needs a better knowledge of the effect that the physicochemical properties of the surroundings and the temperature has onto the aggregation mechanism. Particularly, temperature is perhaps the least understood parameter, mainly because most of the studies have been performed at temperatures below $100 \mathrm{~K},{ }^{31}$ since the number of monomer units required for aggregation increases inversely proportional to the temperature. ${ }^{27}$ Nevertheless, Alfimov et $a l .{ }^{32}$ revealed the arrangement of J-aggregates and dimer formation with different structures and in amorphous dispersions upon photoexcitation and temperature changes. Shapiro and co-workers ${ }^{33}$ have demonstrated temperature dependence results in a mixture of monomer, dimers, and J-aggregates at body temperature, showing different J-aggregate bands linked to the cis- or transconformation. Additionally, Kaiser et al. ${ }^{34}$ reported a temperature dependence study on the aggregation behavior of a perylene derivative associating their observations to the arrangement angle. In order to expand the modest existent understanding of high temperature effects in molecular aggregates, we have recently initiated studies of this effect in a cyanine derivative. 
Herein, we report the ability to control the ratio of $\mathrm{H}$-aggregates versus monomer and/or J-aggregates with heat in a cyanine derivative. A complete experimental temperature study between 23 and $90{ }^{\circ} \mathrm{C}$, on thiacarbocyanine dye (THIATS) molecule (3,3, -disulfopropyl-5,5, -dichloro-9ethylthiacarbocyanine), was performed to investigate its effect on conformational dynamics of THIATS species. We present the theoretical modeling revealing the molecular stacking features that evidence changes in intermolecular conformation with temperature. We discuss the evolution of aggregation in different mixtures containing $\mathrm{H}$-aggregates, J-aggregates, and/or monomer depending on THIATS concentration and conformations.

\section{EXPERIMENTAL METHODS}

THIATS was purchased from Hayashibara Biomedical Laboratories and used without any further purification. All the measurements were carried out in aqueous solution using ultrapure deionized water (the influence from the cell and solvent were previously subtracted). In order to make the monomeric and the $\mathrm{H}$ - and J-aggregates in the solution, four different mixtures of THIATS were prepared at diverse concentrations according to Refs. 16 and 35. It is well known that by increasing the concentration of cyanine dyes in the solution one can gain control on the aggregation process. ${ }^{3}$ Therefore, we prepared a solution of the monomer at a concentration of $0.5 \mathrm{mM}$ (1), H-aggregates at $1 \mathrm{mM}$ (2), a mixture of $\mathrm{H}$-aggregates and J-aggregates at $2 \mathrm{mM}$ (3), and J-aggregates at a concentration of $10 \mathrm{mM}$ (4).

(a) The absorbance measurements were carried out with a single-beam spectrophotometer (Agilent 8453 Diode Array UV-VIS) in quartz cells of different widths depending on the THIATS concentration: $1 \mathrm{~cm}$ thickness for monomer, $1 \mathrm{~mm}$ for H-aggregates, $0.2 \mathrm{~mm}$ for $\mathrm{H} / \mathrm{J}$ aggregates mixtures and, $0.01 \mathrm{~mm}$ for J-aggregates. This system has an operational spectral range of 190$1100 \mathrm{~nm}$ with a resolution of better than $2 \mathrm{~nm}$ and less than $0.03 \%$ stray light. The instrument was computer controlled with Agilent ChemStation software.

(b) The steady-state fluorescence spectrum in water and the excitation anisotropy analogous in poly-THF were measured under the right angle geometry in a $1 \mathrm{~cm}$ quartz cell using a PTI Quanta Master and steady state spectrofluorimeter (model QM-3/2005) equipped with a $75 \mathrm{~W}$ xenon lamp and two QuadraScopic ${ }^{\mathrm{TM}}$ monochromators (excitation and emission), tunable in a wavelength range from 180 to $2000 \mathrm{~nm}$. This instrument has a continuously adjustable spectral bandwidth from 0 to $25 \mathrm{~nm}$ via micrometer slits and stray light $<0.02 \%$.

(c) Temperature dependence experiments were performed using a Peltier system to warm up the different samples. In order to avoid the saturation of the absorption, quartz cells of different widths were used depending on the THIATS concentration. Samples were first heated up to a stable temperature from room temperature $\left(23-90{ }^{\circ} \mathrm{C}\right)$. For each temperature, the absorption spectrum was measured after the thermal equilibrium was achieved in all the solutions (after approximately $20 \mathrm{~min})$.

\section{THEORETICAL APPROACH}

In order to model our system in its monomeric and aggregated forms, GAUSSIAN 03 and GAUSSIAN 09 software were employed. ${ }^{36}$ Because the size of the aggregates to be studied precluded the use of more sophisticated approaches such as density functional theory, a semiempirical model was used to optimize the energy of THIATS. For this dye molecule, dispersion interactions were included with the semiempirical model AM1 (Ref. 37) and IOP (3/124=1) option (denoted $\mathrm{AM} 1+\mathrm{D}$ hereafter), through the use of a simple ad hoc parameterized $\mathrm{r}^{-6}$ function, which is an option available in GAUSSIAN. Although the fragment used in the computation lacked the ions, solvent molecules, and surrounding environment of the crystal, the agreement was still acceptable.

With the aim of computing the electronic excitations of THIATS using the energy optimized structures obtained as described above, the ZINDO-S method ${ }^{38}$ was chosen. Because this approach has not been thoroughly tested for chlorine, this halogen was simply substituted by fluorine in the optimized structures before performing the ZINDO-S calculations.

For the optimization of the geometry of several putative $\pi$-stack structures, we used optimization (Cartesian, loose) that yielded acceptable structures in reasonable amounts of time. Through the employment of this particular approach a significant reduction of the presence of several lowfrequency modes characteristic of weak complexes in which one entire molecule slips or moves with respect to another is possible, thus reducing computing costs.

\section{RESULTS AND DISCUSSION}

\section{A. EXPERIMENT}

In Fig. 1, we show the THIATS molecular structure as an inset and the UV-VIS (visible) absorption, fluorescence, and excitation anisotropy spectra of this dye. The absorption spectrum of THIATS at low concentration $\left(10^{-4} \mathrm{M}\right)$ exhibits the typical monomer band centered at $554 \mathrm{~nm}$ corresponding to the allowed $S_{0} \rightarrow S_{1}$ transition. This transition is confirmed by the remarkable plateau in the excitation anisotropy spectrum between 520 and $560 \mathrm{~nm}$. The evident decrease in the values of excitation anisotropy at shorter wavelengths indicates the formation of $\mathrm{H}$-aggregates. According to Chibisov and co-workers ${ }^{28}$ and Takahashi et al., ${ }^{16}$ the shoulder observed at $514 \mathrm{~nm}$ in the absorption spectrum is attributed to the presence of H-aggregates. However, our theoretical calculations revealed that this band is more in agreement with the presence of and $\mathrm{H}$-tetramer $(\mathrm{H}-4)$ as explained later in Sec. IV B. The fluorescence spectrum shown in Fig. 1 suggests, through the presence of an only narrow band with maximum at approximately $570 \mathrm{~nm}$ that the radiative decay is only produced by the monomer.

To understand the temperature effect on the formation of different aggregate species in THIATS, we first prepared four 


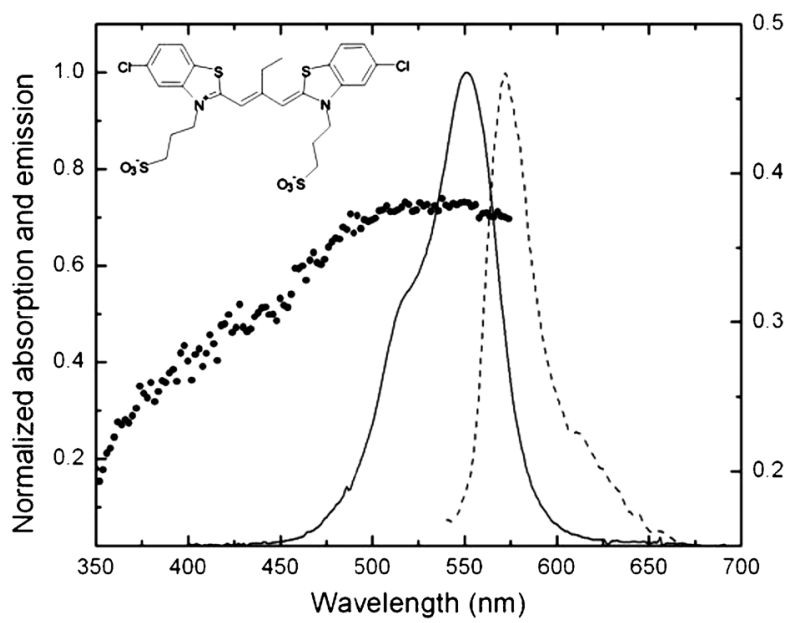

FIG. 1. Absorption (solid), fluorescence (dash), and excitation anisotropy spectra (dots) of a THIATS/water solution at a concentration of $0.5 \mathrm{mM}$. The inset displays the molecular structure of THIATS.

solutions of this dye at different concentrations according to the literature ${ }^{16,35}$ to corroborate the presence of all the different species subject to our study. The experimental absorption spectra profile of these solutions is then compared with the different theoretical stacking geometries predicted by our modeling. This allowed us to propose a thermal switching mechanism in this specific system. Figure 2 displays the different absorption spectra of all four solutions. The observed characteristic bands exhibited by the different spectra are in good agreement with results reported in Ref. 35: monomer band at $554 \mathrm{~nm}, \mathrm{H}-4$ at $514 \mathrm{~nm}$, and J-aggregates at $624 \mathrm{~nm}$. The shoulder on the blue side of the spectra in the solution (3) is attributed as explained before to a higher degree of aggregation, i.e., an $\mathrm{H}-4 .^{2,39}$

Figure 3 portrays the temperature effect on the conformational dynamics of the different aggregate solutions studied. Particularly, Fig. 3(a) shows the results obtained for solution (2) (H-aggregate/monomer mixture). At $23{ }^{\circ} \mathrm{C}$, the absorbance band of the H-4, centered at $514 \mathrm{~nm}$, is twofolds more intense than that of the monomer band with maximum at $554 \mathrm{~nm}$. As the temperature is augmented (curves 1 to 8 ) the $\mathrm{H}-4$ band vanishes and the monomer band increases. These results are in perfect agreement with those reported by Scheblykin and co-workers ${ }^{40}$ and are attributed to an entropic factor. In accordance to Ref. 40, the heating process induces a dissociation of the $\mathrm{H}$-aggregates into a monomer unpacking the monomer units. The linear relationship between the H-4 and the monomer and, the absence of other species in this solution at different temperatures, is confirmed by the existence of only the two bands described above and the presence of an isosbestic point around $533 \mathrm{~nm}$. In fact, this point is observed in all solutions where the monomer and $\mathrm{H}-4$ coexist. At $50{ }^{\circ} \mathrm{C}$, one can notice that the two bands, corresponding to the two different species in the solution, present virtually the same absorbance. Raising the temperature even further, the identified trend between the two bands persists. In fact, at $90{ }^{\circ} \mathrm{C}$, nearly all THIATS is in the form of monomer. Interesting to highlight is the fact that

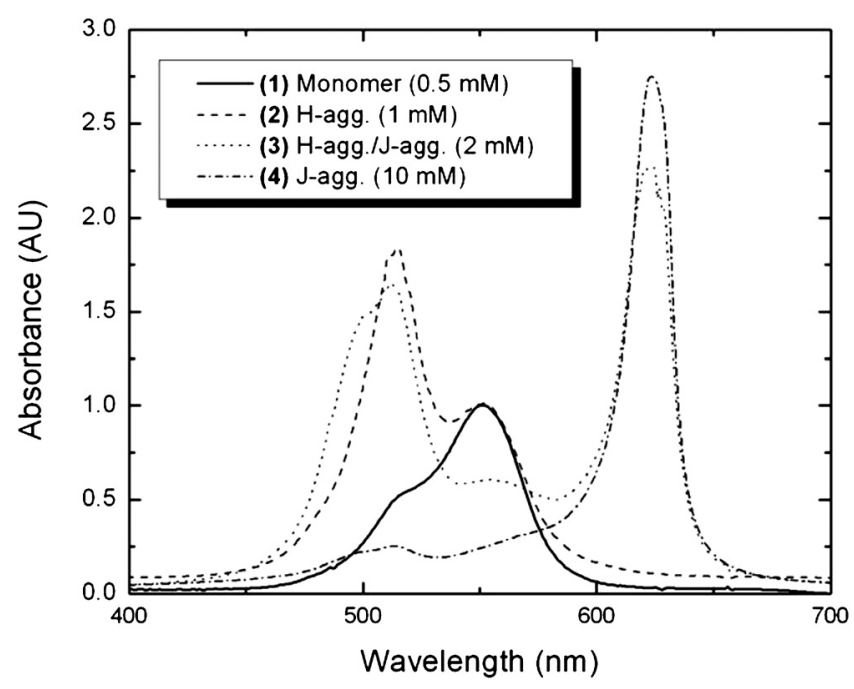

FIG. 2. Absorbance spectra of THIATS aqueous solutions taken at four different concentrations: (1) monomer [0.5 mM], (2) H-aggregate and monomer [1 mM], (3) H-aggregate, monomer, and J-aggregate [2 $\mathrm{mM}]$, and (4) J-aggregate $[10 \mathrm{mM}]$.

up to a maximum temperature of $80{ }^{\circ} \mathrm{C}$ the process is totally reversible by decreasing the temperature within a relative short period of time (approximately $5 \mathrm{~min}$ ).

Subsequently, the temperature dependence study of solution (3) was performed with the aim to understand the behavior of a mixture of monomer, $\mathrm{H}$ - and J-aggregates [Fig. 3(b)]. According to our theoretical calculations, these two bands correspond to $\mathrm{H}$ - and J-tetramers, $\mathrm{H}-4$ and J-4, respectively (see Sec. IV B). In this solution, we observed an increase in the monomer band at expenses of a fast and total depletion of the J-aggregates band, between 40 and $50{ }^{\circ} \mathrm{C}$. Moreover, another isosbestic point at $569 \mathrm{~nm}$ was detected between these two species in the solution. At temperatures as high as $80^{\circ} \mathrm{C}$, the absorption spectrum of the THIATS solution resembles that at a concentration of $1 \mathrm{mM}$ presented in Fig. 3(a) containing monomer and $\mathrm{H}$-aggregates (room temperature). This indicates that J-aggregates break apart leaving room for the formation of only $\mathrm{H}$-aggregates and monomer. At $90{ }^{\circ} \mathrm{C}$, the $\mathrm{H}$-aggregate spectrum shows a little decrease while the monomer band increases. This result is in agreement with those presented at lower concentration [solution (2)]. Indeed, the monomer absorbance is twice the initial one (see Fig. 1). To recover the room temperature spectrum, with a preponderance of J-aggregates one should wait approximately $15 \mathrm{~min}$. The slow recovery process is an indication of the memory effect present in the mixture of $\mathrm{H}$ - and J-aggregates, as well as monomer in the solution. Therefore, controlling the dominant species in the solution as a function of temperature as well as time is a possibility.

Similar type of experiments was performed in solution (4) [see Fig. 3(c)]. One can confirm the presence of an overwhelming amount of J-aggregates over the other two species in the mixture. An increase in temperature up to $50{ }^{\circ} \mathrm{C}$ changes slowly, but not dramatically, the ratios J-aggregates/ $\mathrm{H}-4$ and J-aggregates/monomer. However, for a greater change in temperature, the $\mathrm{H}-4$ and monomer bands increase whereas the J-aggregate band decreases. A total depletion of 

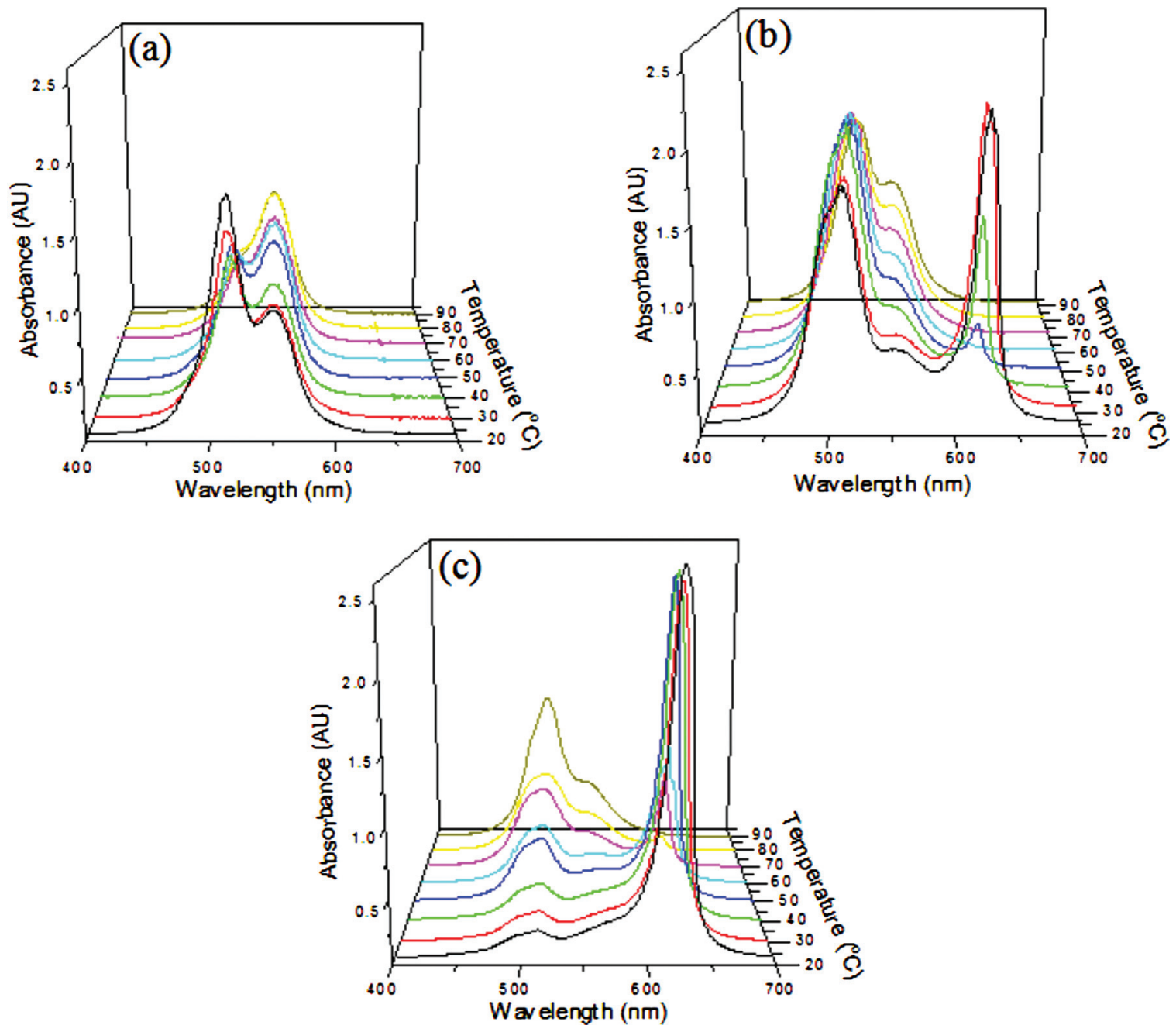

FIG. 3. 3D plots of the absorption spectra of THIATS as a function of temperature: (a) solution (2), (b) solution (3), and (c) solution (4).

the latter is achieved at $90{ }^{\circ} \mathrm{C}$ in which case an analog spectrum to that depicted for THIATS in solution (1) (see Fig. 1). A full recovery of the J-aggregate spectrum, observed at room temperature, is possible after $1 \mathrm{~min}$. However, 95\% recovery of the initial J-aggregate spectrum happens with the first $15 \mathrm{~s}$.

Based on our experimental results, we propose a thermal switching mechanism between the different species of THIATS in a solution to summarize their conformational dynamics (Fig. 4). In general, all the reactions proposed are reversible or present a "memory effect," that is, the monomer or monomer/aggregate species obtained by increasing the temperature (forward reaction) can be reinstated to their original aggregation state [solutions (2)-(4)] by lowering the temperature back to room temperature and storing them for different time periods (reverse reaction). Particularly, solution (2) (Fig. 4, top), comprising of the H-4 and monomer, is shifted toward the monomer, which, under these conditions, exist at a concentration that is twofold higher than that of solution (1). On the other hand, solution (3) (Fig. 4, middle), consisting of a mixture of $\mathrm{H}-4, \mathrm{~J}-4$, and monomer, is converted upon heating to an $\mathrm{H}-4 /$ monomer mixture, similar to the case of solution (4) (Fig. 4, bottom) that contains J-4 aggregates. These different species that result from heating can be taken back to their original state in 5,15 , and $1 \mathrm{~min}$, respectively. The fact that solution (3) takes the longest to reach its original state implies a more complex dynamics between the three species involved in the solution. In all cases, higher concentrations of THIATS monomer compared to the regular concentrations of this species can be obtained with an increase in temperature. This might be of potential benefit for applications in which a high concentration of monomer is desired without the formation of the corresponding aggregated species.

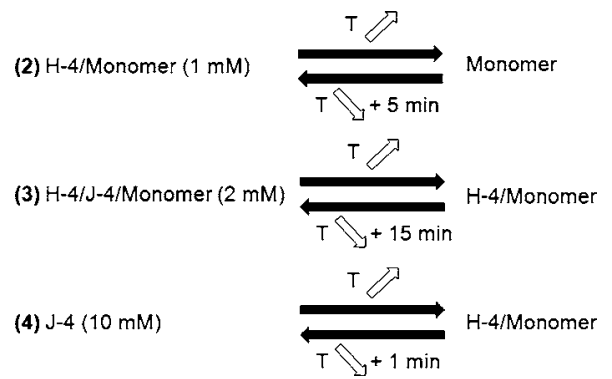

FIG. 4. Synopsis of the proposed temperature switching mechanisms on THIATS molecular aggregates at different concentrations: $1 \mathrm{mM}(2), 2 \mathrm{mM}$ (3), and $10 \mathrm{mM}(4)$. 


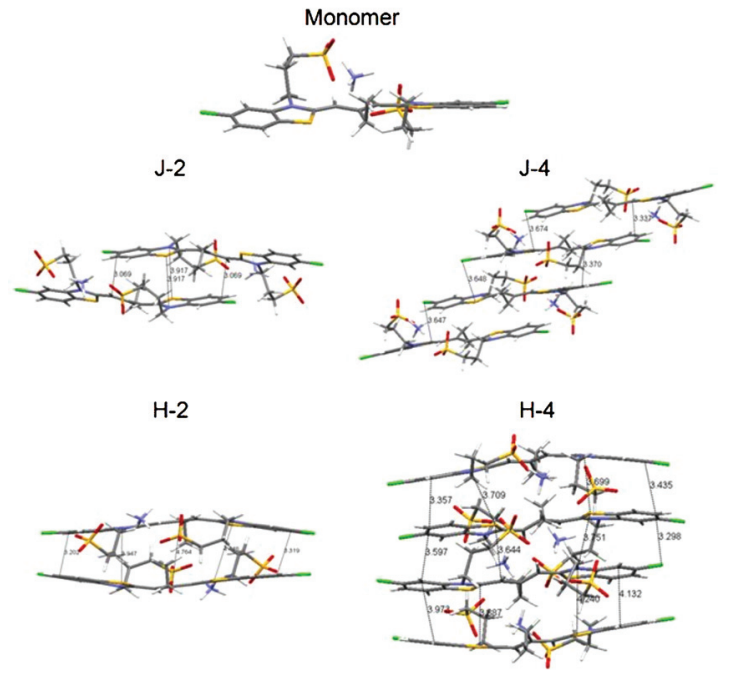

FIG. 5. Representation of THIATS stacking configurations in the energy optimized geometries using AMD+1 model: monomer, J-2, J-4, H-2, and $\mathrm{H}-4$.

\section{B. THEORY}

In order to explain our experimental results, the theoretical calculations on THIATS in its monomeric and aggregated forms were systematically performed. As stereo chemical possibilities are not expected to have very different electronic spectra, ${ }^{41}$ the single monomeric isomer shown in Fig. 5 was selected as the repeat unit in aggregates. The computed absorption of the AM1 +D energy-optimized monomeric structure in water was found to be in acceptable agreement with the experimental value $(556 \mathrm{~nm}) .{ }^{42}$ Figure 5 also illustrates the geometry adopted for the ion-counterion association.

The modeling of aggregates was performed based on McRae-Kasha rules ${ }^{43}$ that are derived to take into account the interaction of transition dipoles associated with the main single photon transition of a single unit, with those of other units in the stack. Spectral blue- or redshifts resulted from differences in the stacking geometry. In our case, the stacking configuration was characterized by a single variable, $\alpha$, the angle between the long axis of the cyanine monomer rings and the translational vector that carries one asymmetric unit to the next in the aggregate. Values of $\alpha$ larger than approximately $55^{\circ}$ were predicted to result in blueshifts $(\mathrm{H}$ stacks), while redshifts (J stacks) result from lesser values. ${ }^{2}$

Because a considerable number of possible $\mathrm{H}$ and $\mathrm{J}$ stacks can be constructed by inverting and then translating the optimized monomer geometry, inversion was then used to keep the ionic chains apart from one another on adjacent molecules. Thus, every molecule was inverted with respect to its nearest neighbor as one travels either horizontally or along the translation vector of the stack. This approach happened to allow the $\pi-\pi$ interaction to determine the stacking geometry.

Using the AM1 +D energy-optimized structure, we were able to construct the best J-dimers (J-2) and J-tetramer (J-4) structures, as shown in Fig. 5. Furthermore, we calculated their corresponding absorption spectra within the visible (Fig. 6). While the absorption spectrum of J-2 showed a red-

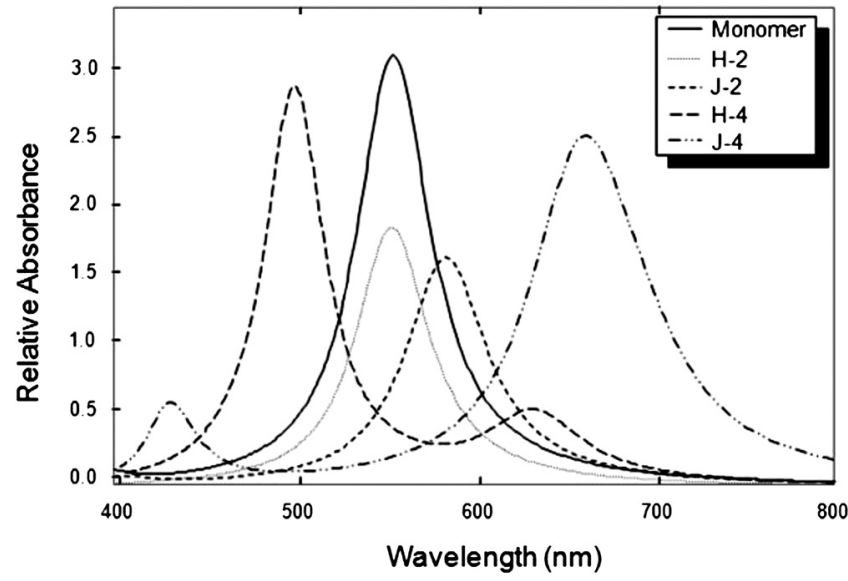

FIG. 6. Theoretical relative absorption spectra of monomer, H-2, H-4, J-2, and J-4 calculated with ZINDO-S method.

shift band centered at $\lambda_{\max }=584 \mathrm{~nm}$ that of the J-4 aggregate is centered at $659 \mathrm{~nm}$. Through the comparison of these results with the experimental spectrum for the J-aggregates $(624 \mathrm{~nm})$, we conclude that the J-4 is the preferred configuration in an aqueous solution of THIATS. Other J-aggregates with a different number of units and similar arrangement were also tested but the absorption bands were not as close to the experimental value as J-2 and J-4. Therefore they were discarded from the analysis.

Next, the $\mathrm{H}$ stacks were examined according to the packing arrangement (see Fig. 5). Out of multiple possibilities, it was found that while dimers have a single absorption peak at $\lambda_{\max }=558 \mathrm{~nm}$, i.e., in close proximity to the monomer band $(556 \mathrm{~nm})$, the computed spectrum of H-4 exhibits two distinct maxima, an intense one at $500 \mathrm{~nm}$ and a weaker one at $632 \mathrm{~nm}$ (Fig. 6). The reproduced intense absorption band in $\mathrm{H}-4$ is in good agreement with the experimental results obtained for the same aggregates (see Fig. 2).

Having established the preferential stacking arrangement on THIATS and knowing that energy values can be used for intercomparison of the various structures, the heat of formation at standard conditions was calculated through the parameterization of AM1 in order to gain some insight on the temperature dependence effect reported herein.

Through a direct comparison of the calculated values for the $\mathrm{H}$ and $\mathbf{J}$ stacks of the same number of monomeric units, we found the former to always be lower in energy. This indicates that this type of aggregates in THIATS is thermodynamically favored. This result is in good accordance with the role of dispersion interactions in the stabilization of these structures as a result of the elevated number of close contacts in the $\mathrm{H}$ structures, in contrast to the J. In addition, this outcome is supported by the larger derived $\pi$-interaction energy of $\mathrm{H}$ aggregate structures. The observed irregularities, although in this configuration, are perhaps, the result of possible steric interactions not very important in the $\mathrm{J}$ aggregate. The difference in stability for the dimmers is in the range between 4 and $17.6 \mathrm{kcal}$ while for the tetramers is approximately $62.2 \mathrm{kcal}$. One possible explanation for such a large difference is the apparent dissimilarity in the solvation sphere around the different aggregates. As mentioned in the 
foregoing, the solvation effects on the various structures are expected to be a significant factor in describing the formation of the molecular stacks. Proof of this hypothesis is the favored packing in water but not in aprotic solvents such as DMSO, DMF, and others similar liquids without a predominant admixture of $\mathrm{H}_{2} \mathrm{O}$. As a further examination of our results, the energies of the tetramer stacks were computed using the SCRF (SMD) model in water as solvent. The resulting energy difference between $\mathrm{H}-4$ and $\mathrm{J}-4$, equal to $48.6 \mathrm{kcal}$, is smaller than that previously reported but it is still large enough to pose an essentially insurmountable barrier for an $\mathrm{H} \rightarrow \mathrm{J}$ transformation at room temperature. This large difference between the tetramers is surprising in light of the established formation of $\mathrm{J}$ aggregates. A possible mechanism for the formation of the $\mathrm{J}$ stacks is that the energetically favored $\mathrm{H}$ stacks are formed in a shorter time scale, i.e., they are kinetically favored. Therefore, $\mathrm{H}$ aggregates may or may not form beyond the tetramer configuration. Nevertheless, slipping of the various levels could promote a molecular rearrangement that could lead to J stacking. In addition, reduction of the energy difference between $\mathrm{H}$ and $\mathrm{J}$ motifs might occur upon the formation of two-dimensional (2D) sheets in which the long axis of the cyanines are all parallel. The energy difference between the two motifs under discussion is expected then to be smaller than that between one-dimensional stacks because there is a proportionately less projected between the levels. Such overhang would be produced in an $\mathrm{H} \rightarrow \mathrm{J}$ transformation by a loss of close contact between levels and concomitant reduction of the dispersion interactions. Another explanation is the fact that large cation-anion interaction energies can alter the side-chain geometries, thus lowering the energy difference between the two types of aggregates and permitting the facile $\mathrm{H}$ to $\mathrm{J}$ conversions. Such a transformation would be most likely in a 2D sheet as mentioned before where differences in edgeeffects are minimized. Further studies are in progress.

\section{CONCLUSION}

We have experimentally demonstrated the possibility of thermal switching of aggregates in a cyanine dye. This novel approach provides control on the preferential species desired in the solution at concentrations never attempted before. We have demonstrated the possibility of preparing THIATS monomers solutions at a concentration 100 times greater that of the most likely for its presence at standard conditions. An agreement between experiments and theory has made possible the identification of the actual size and type of aggregate present in THIATS solution at different concentrations and temperatures. Through the use of this tactic we envisage new applications of this principle in biology and medicine, where concentration and aggregation is a crucial factor to be considered.

\section{ACKNOWLEDGMENTS}

This work was supported by the National Science Foundation (Contract No. CHE-0832622).
${ }^{1}$ E. E. Jelley, Nature (London) 138, 1009 (1936); G. Scheibe, Angew. Chem. 49, 567 (1936).

${ }^{2}$ E. S. Emerson, M. A. Conlin, A. E. Rosenoff, K. S. Norland, H. Rodriguez, D. Chin, and G. R. Bird, J. Phys. Chem. 71, 2396 (1967).

${ }^{3}$ A. H. Herz, Adv. Colloid Interface Sci. 8, 237 (1977).

${ }^{4}$ T. Hoshi, H. Saiki, S. Kuwazawa, C. Tsuchiya, Q. Chen, and J. Anzai, Anal. Chem. 73, 5310 (2001).

${ }^{5}$ W. J. Dulmage, W. A. Light, S. J. Marino, C. D. Salzberg, D. L. Smith, and W. J. Staudenmayer, J. Appl. Phys. 49, 5543 (1978).

${ }^{6}$ K. Saito, J. Phys. Chem. B 103, 6579 (1999).

${ }^{7}$ B. Becker, J. Clapper, K. R. Harkins, and J. A. Olson, Anal. Biochem. 221, 78 (1994).

${ }^{8}$ M. Reers, T. W. Smith, and L. B. Chen, Biochemistry 30, 4480 (1991).

${ }^{9}$ S. Das and P. V. Kamat, J. Phys. Chem. B 103, 209 (1999).

${ }^{10}$ F. Meng, K. Chen, and H. Tian, Appl. Phys. Lett. 82, 3788 (2003).

${ }^{11}$ N. Miyamoto and K. Kuroda, J. Am. Chem. Soc. 123, 6949 (2001).

${ }^{12}$ W.-J. Wu, W.-H. Zhan, J.-Li. Hua, and H. Tian, Res. Chem. Intermed. 34, 241 (2008).

${ }^{13}$ J. Franck and E. Teller, J. Chem. Phys. 6, 861 (1938).

${ }^{14}$ J. Frenkel, Phys. Z. Sowjetunion 9, 158 (1936).

${ }^{15}$ M. Liu, A. Kira, and H. Nakahara, J. Phys. Chem. 100, 20138 (1996).

${ }^{16}$ D. Takahashi, H. Oda, T. Izumi, and R. Hirohashi, Dyes Pigm. 66, 1 (2005).

${ }^{17}$ W. West and S. Pearce, J. Phys. Chem. 69, 1894 (1965).

${ }^{18}$ T. D. Slavnova, H. Görner, and A. K. Chibisov, J. Phys. Chem. B 111, 10023 (2007).

${ }^{19}$ A. K. Mandal and M. K. Pal, Chem. Phys. 253, 115 (2000).

${ }^{20}$ W. J. Harrison, D. L. Mateerb, and G. J. T. Tiddy, Faraday Discuss. 104, 139 (1996).

${ }^{21}$ N. Vranken, M. Van der Auweraer, F. C. De Schryver, H. Lavoie, P. Belanger, and C. Salesse, Langmuir 16, 9518 (2000).

${ }^{22}$ A. K. Chibisov, V. Prokhorenko, and H. Gorner, Chem. Phys. 250, 47 (1999).

${ }^{23}$ A. V. Vannikov, A. D. Grishina, B. I. Shapiro, L. Y. Pereshivko, T. V. Krivenko, V. V. Savelyev, V. I. Berendyaev, and R. W. Rychwals, Chem. Phys. 287, 261 (2003).

${ }^{24}$ N. Wang, A. A. Muenter, and S. Mukamel, J. Chem. Phys. 99, 3604 (1993).

${ }^{25}$ K. Minoshima, M. Taiji, K. Misawa, and T. Kobayashi, Chem. Phys. Lett. 218, 67 (1994).

${ }^{26}$ I. D. L. Albert, T. J. Marks, and M. A. Ratner, J. Phys. Chem. 100, 9714 (1996).

${ }^{27}$ J-Aggregates, edited by T. Kobayashi (World Scientific, Singapore, 1996).

${ }^{28}$ A. K. Chibisov, G. V. Zakharova, and H. Görner, Phys. Chem. Chem. Phys. 1, 1455 (1999); I. G. Shcheblykin, A. Y. Sliusarenko, N. N. Bataev, M. Van der Auweraer, O. P. Varnavsky, and A. G. Vitukhnovsky, J. Russ. Laser Res. 20, 7 (1999); I. G. Scheblykin, M. M. Bataiev, M. Van der Auweraer, and A. G. Vitukhnovsky, Chem. Phys. Lett. 316, 37 (2000).

${ }^{29}$ E. Gaizauskas and K.-H. Feller, Opt. Commun. 216, 217 (2003).

${ }^{30}$ G. V. Zakharova and A. K. Chibisov, High Energy Chem. 43, 294 (2009).

${ }^{31}$ H. Fidder, J. Knoester, and D. A. Wiersma, Chem. Phys. Lett. 171, 529 (1990); V. F. Kamalov, I. A. Struganova, and K. Yoshihara, J. Phys. Chem. 100, 8640 (1996); T. E. Kaiser, I. G. Scheblykin, D. Thomsson, and F. Würthner, J. Phys. Chem. B 113, 15836 (2009).

${ }^{32}$ M. V. Alfimov, A. A. Shtykova, and V. F. Razumov, High Energy Chem. 40, 18 (2005)

${ }^{33}$ B. I. Shapiro, E. A. Belonozhkina, and V. A. Kuz'min, Nan. Russ. 4, 38 (2009).

${ }^{34}$ T. E. Kaiser, H. Wang, V. Stepanenko, and F. Würthner, Angew. Chem., Int. Ed. 46, 5541 (2007).

${ }^{35}$ H. Yao, K. Domoto, T. Isohashi, and K. Kimura, Langmuir 21, 1067 (2005).

${ }^{36}$ M. J. Frisch, G. W. Trucks, H. B. Schlegel et al., GAUssian, Gaussian, Inc., Wallingford, CT, 2009.

${ }^{37}$ M. J. S. Dewar, E. G. Zoebisch, E. F. Healy, and J. J. P. Stewart, J. Am. Chem. Soc. 107, 3902 (1985). 
${ }^{38}$ J. Ridley and M. Zerner, Theor. Chim. Acta 32, 111 (1973).

${ }^{39}$ W. West, S. P. Lovell, and W. Cooper, Photograph. Sci. Eng. 14, 52 (1970).

${ }^{40}$ I. G. Scheblykin, O. P. Varnavsky, W. Verbouwe, S. DeBacker, M. Van der Auweraer, and A. G. Vitukhnovsky, Chem. Phys. Lett. 282, 250
(1998).

${ }^{41}$ J. T. Knudtson and E. M. Eyring, J. Phys. Chem. 78, 2355 (1974).

${ }^{42}$ C. Toro, L. De Boni, S. Yao, J. P. Ritchie, A. E. Masunov, K. D. Belfield, and F. E. Hernandez, J. Chem. Phys. 130, 214504 (2009).

${ }^{43}$ E. G. McRae and M. Kasha, J. Chem. Phys. 28, 721 (1958). 